\title{
Methanogenic Granules Are Replicated, Whole Microbial Communities With Reproducible Responses To Environmental Cues
}

\section{Anna Christine Trego}

National University of Ireland Galway

\section{Sarah O'Sullivan}

National University of Ireland Galway

Simon Mills

National University of Ireland Galway

\section{Estefania Porca}

National University of Ireland Galway

\section{Christopher Quince}

University of Warwick Warwick Medical School

\section{Umer Zeeshan ljaz}

University of Glasgow

Gavin Collins ( $\sim$ gavin.collins@nuigalway.ie )

National University of Ireland - Galway https://orcid.org/0000-0002-9947-1130

\section{Research}

Keywords: Anaerobic digestion; biofilms; methanogens; microbial communities; sludge granules; wastewater.

Posted Date: March 12th, 2020

DOI: https://doi.org/10.21203/rs.3.rs-16883/v1

License: (c) (i) This work is licensed under a Creative Commons Attribution 4.0 International License. Read Full License 


\section{Abstract}

Background In this study, individual anaerobic granular biofilms were used as true community replicates to assess whole-microbial-community responses to environmental cues. The aggregates originated from three different biomass sources, i.e. three different engineered biological wastewater treatment systems, were each size-separated into three fractions - small, medium and large - and characterised according to organic matter concentrations and rates of methanogenic activity. Differences in the microbial community structure of each size fraction from each source were determined using 16S rRNA gene sequencing. Subsequently, single granules from the large size fraction of one of the sources were separately subjected controlled environmental cues in novel micro batch reactors (mBRs). Results Organic content, methanogenic activity, and microbial community were significantly different between the three size fractions, with diversity trajectories replicated across the three sludge sources - indicating a potential development model as granules age. Individual large granules from one of these sources were statistically identical with respect to the structure of the active community based on cDNA analysis. It was observed that the active microbial community of individual granules, at the depth of 16S rRNA sequencing, produced reproducible responses to environmental conditions. While each condition resulted in the up-regulation of particular OTUs and clades, the core microbiome, consisting of many fermentative bacteria along with methanogenic archaea, namely, Methanosarcina and Methanobacterium , persisted. Conclusions At this level, single anaerobic granules can be considered highly-replicated wholeecosystems, opening the door to high-throughput studies in Microbial Ecology.

\section{Background}

Microbial communities underpin biogeochemical cycles of Earth's soil, oceans, and atmosphere, providing ecosystem services and functions for plants, animals, and humans. However, our understanding of their structure-function relationships is highly limited. Recent studies studies have refined increasingly innovative approaches to study spatio-temporal phylogenetic and functional dynamics of environmental microbiomes [1] - a challenge requiring creative solutions. A pressing challenge will be to determine, and model, the response of microbial communities to environmental change, including changing climates. To achieve this, whole microbial communities must be reliably sampled, or at least reliable replicates are required from communities [2]. Indeed, the complexity of the physico-chemical matrices in which natural microbiomes are typically embedded complicates the task of sampling. The soil matrix, for example, comprises of micro-environments with localised gradients of $\mathrm{pH}$, oxygen concentrations, and redox potential [3]. Equally, the spatial continuum of microbiomes in such environments - not knowing where communities 'start' or 'finish' - further constrains reliable sampling.

Biotechnological applications, in which mixed-species consortia are applied for specific functions, may provide useful model systems, having clear functional parameters and offering controlled environments for sampling [4]. An important feature of many microbial communities is aggregation, not only into biofilm slimes but structured granules, and several important examples are seen across the natural and built environment, including methanogenic, aerobic, annamox, phototrophic and hydrogenic granular 
biofilms in wastewater treatment technologies [5], as well as the pink berry [6] and 'lake ball' [7] granules observed in natural systems. Such aggregates are robust and technologically advantageous over planktonic communities, or even biofilm slimes, due to better retention of cells; protection of some organisms against local environmental changes; and the enmeshment of species supporting mutualistic and syntrophic relationships underpinning otherwise thermodynamically impossible reactions [8]. In those respects, architecturally organized granules, and the granulation phenomenon [9], are fascinating and support the type of interdependent relationships that underpin a variety of modern environmental biotechnologies.

Methanogenic granules, in particular, harbour consortia of multiple microbial trophic groups collectively pushing, and funneling, carbon through successive pathways towards production of biogas or other highvalue intermediates [10], facilitating wastewater treatment and biorefinery innovations. Indeed, this is facilitated by the highly organised spatial distribution in methanogenic granules of the various trophic groups [11]. In the same way that soil aggregates can be considered as whole communities and an important consideration with respect to 'fine-scale' structure of the microbial community [3], methanogenic aggregates may be equally important for community ecology inside built ecosystems.

For those reasons, interest in the ecology of granules and other aggregates is expanding beyond applications in engineered systems. Recently, Leventhal et al. [12] used aerobic granules as replicated communities to investigate the extent to which intrinsic or extrinsic factors influence microbiome structure - and at which degree of taxonomic resolution community types can be resolved. Clear community types were not observed at the genus-level, where community replicates were highly varied, but were apparent at strain-level, indicating strain-level diversification [12]. Further characterisation of single granules will be useful through a community-assembly lens, but data in the literature are limited to studies of $16 \mathrm{~S}$ rRNA genes [13]. This leaves an opportunity for microbial ecologists to lever granular biofilms as whole microbial communities, in a range of compelling ways to address pressing questions on the structure-function of complex microbiomes.

In this study RNA from individual methanogenic granules was studied to determine the impact of source and size on the structure of the active fraction of the microbiome. The impact of a range environmental cues on the active microbiomes was then investigated using single-granule micro-batch reactor incubations. The questions posed by this study have implications for conceptual breakthroughs in developing 'high-throughput' single-ecosystem Microbiology. The experiments offer an example of how whole communities may be easily monitored, toward models predicting responses to environmental cues.

\section{Results}

\section{Physiological characterisation across source and size}

The size distribution, based on separation into small $(S)$, medium $(M)$, and large $(L)$ size fractions, was statistically similar $(p=0.287)$ between the three different community sources. For each source, the $L$ size fraction dominated, and made up over $50 \%$ of the size distribution (Fig S1). No significant difference 
$(p=0.054)$ in total solids (TS; $m / v)$ concentrations was found according to source, however, significant differences $(p=0.036)$ were observed between the different size fractions. In general, TS increased with granule size. Significant differences were observed for volatile solids (VS) according to both source ( $p=$ $0.013)$ and size fraction $(p=0.05)$, where $M$ sizes consistently contained the highest VS concentrations. Moreover, individual $L$ granules from each source were found to have statistically similar TS $(p=0.457)$ and VS $(p=0.635)$ values.

In general, regardless of community source or size fraction, specific methanogenic activity (SMA) assays indicated higher methanogenic activities against hydrogen, as opposed to acetate (Fig S1). No significant difference was observed in hydrogenotrophic activity between Source 1 and Source $2(p=0.168)$, but hydrogenotrophic activity of Source 3 was significantly different to the other two sources $(p=0.000)$. Furthermore, significant differences were found between $M$ and $L$ size fractions, which generally had higher activities, overall.

\section{Phylogenetic differences across granule source and size}

Community source was found to be the most significant driver of microbial community structure of individual granules, within which internal differences were driven by granule size (Fig 1). Alpha and beta diversity analyses revealed that samples clustered strongly according to source, with smaller gradients according to size. PERMANOVA verified that both community source and size fraction were significant factors shaping the microbiome of single granules, however, source was consistently a stronger driver of the microbial community structure than size. For example, PERMANOVA using the Bray-Curtis metric determined that source was responsible for $66 \%$ of the variation in the microbiome between samples $(p=$ $\left.0.001^{\star * *}\right)$, while size was only responsible for $4 \%$ of the variation $(p=0.004 * *)$. Source also played a large role on the phylogenetic alpha diversity (environmental filtering) - where Sources $1 \& 2$ had a more evenly-spread community, the community making up Source 3 was significantly more phylogenetically clustered. Generally, across all community sources, as size increased, alpha diversity decreased, according to Shannon Entropy, while the phylogenetic alpha diversity increased. Across all sources, the relative abundances of methanogenic archaea were high, including Methanobacterium, Methanosaeta, Methanosarcinaceae and Methanolinea.

Multi-variate integration (MINT) study-wise discriminant analysis used two components to identify only 10 discriminating genera which were solely responsible for the diversity differences across community source and size (Fig 2). UPGMA clustering sorted the samples almost perfectly by source, indicating a high similarity between discriminant abundance of each source. The ten discriminants include bacterial genera such as Brachymonas, Variovorax, Propioniclava, Thauera, Brooklawnia, Anaerolinea, Georgenia, Sedimentibacter, Fusibacter and Acetobacterium. No methanogenic archaea were determined to be discriminating across the three community sources and according to size, indicating that they are part of a core anaerobic digestion (AD) microbiome, which statistically, is present in all AD granules, and across the sizes. 
One-way subject ANOVA determined that sixteen individual granules, from a single community source (Source 3 ) and one size fraction (L) had statistically identical active microbiomes $(p>0.5)$. The active community structure was made up of a majority of methanogenic archaea belonging to the phylum Euryarchaeota (Fig 3). In fact, over $50 \%$ of the active community structure comprised Methanobacterium, Methanosarcinaceae, and Methanosaeta.

Targeted metabolomics of single granules incubated under various environmental parameters

pH Effect: Granules incubated at pH 7 showed the most complete volatile fatty acid (VFA) degradation capacity (Fig S2). Residual VFA were observed when incubated at pH 4 and 10. The addition of 2bromoethanesulfonate (BES) as a methanogenic inhibitor had no significant effect on the VFA profile at $\mathrm{pH} 7(\mathrm{p}=0.482), 4(\mathrm{p}=0.716)$ or $10(\mathrm{p}=0.802)$.

Temperature Effect: Lower temperatures yielded reduced rates of VFA consumption (Fig S2). At $37^{\circ} \mathrm{C}$, $91 \%$ of the acetic acid was consumed during the batch period, while reduced temperature conditions decreased the total consumption of acetic acid to $63 \%$. The addition of BES had no significant impact ( $p$ $=0.148$ ) on VFA profiles at $23^{\circ} \mathrm{C}$.

Cobalt Effect: Cobalt-deprived conditions showed decreased rates of VFA utilisation (Fig S2) compared to cobalt-supplied conditions. The most pronounced effect was on acetic acid which was significantly $(p=$ 0.043) under-utilised when cobalt was not supplied.

Substrate Effect: Acetate-supplied granules produced a significantly different $(p=0.008)$ acetic acid profile to granules supplied with the VFA mixture. Furthermore, glucose-supplied granules had significantly ( $p=0.002)$ increased fermentative rates than granules supplied with cellulose (Fig S3). The addition of BES, to either substrate, had no significant effect on substrate consumption $(p=0.899)$.

\subsection{Response of whole microbial communities to specific environmental cues}

Incubated under a range of environmental conditions, the active community comprising these single granules responded strongly (Fig 3 ) and reproducibly (Fig 4). All conditions resulted in a decrease in rarefied richness - and while BES had no significant effect on substrate uptake rates, the methanogenic inhibitor did produce a significant change in diversity of the active microbiome (Fig 3). The application of environmental conditions to the single granules increased both the net relatedness index (NRI) and nearest taxa index (NTI) compared to the original 16 replicates - indicating increased environmental pressure, deterministically altering the phylogenetic alpha diversity of the active community. $\mathrm{pH}$ conditions, for example produced a strong effect $(p<0.001)$ on the NRI, especially under $\mathrm{pH} 4$ and 10 . Specific taxa also shifted in relative abundance with the changing conditions (Fig 4). Moreover, specific clades became up-regulated under the range of conditions (Fig 5), however, the core microbiome, including many of the methanogenic archaea, remain stable.

Each condition, resulted in an active community which had diversified far beyond the original. Under each of the environmental cues, sparse projection to latent structure (sPLS) discriminant analysis identified 
several discriminating genera, which were responsible for the diversity shifts induced by the prescribed environmental parameters (Fig 4) and heat trees identified particular clades which were up-, or downregulated in response (Fig 5).

The $\mathrm{pH}$ conditions produced 27 discriminating genera which were either significantly up- or downregulated depending on $\mathrm{pH}$ conditions when compared to the original. These included Methanosaeta, which were significantly down-regulated when any change in $\mathrm{pH}$ was induced, Pseudomonas, Mesotoga and $\mathrm{Ca}$. Soleaferrea, which were upregulated under high $\mathrm{pH}$ conditions $(\mathrm{pH} 10)$, Petrimonas, Citrobacter and Arcobacter, which were up-regulated under low pH conditions $(\mathrm{pH} 4)$ and finally, Methanosarcina which was up-regulated at $\mathrm{pH} 4,7$ and 10, but down-regulated with the addition of BES.

The provision of various substrates also produced a strong effect on the active community structure. Supplementation with acetate resulted in the up-regulation of Methanosarcina, Achromobacter, Desulfovibrio and Paludibacter, among others. Supplementation of cellulose as the substrate, resulted in the significant down-regulation of taxa such as Tricococcus, Methylomonas, Smithella and Methanosaeta, among others, while Methanosarcina, Nitrosomonas, Citrobacter and Mangroviflexus were up-regulated. Supplementation with the VFA mixture resulted in the up-regulation of Petrimonas,

Citrobacter and Arcobacter, and finally, supplementation with glucose also resulted in the down-regulation of Smithella and Longilinea, but the up-regulation of taxa such as Ca. Soleaferrea, Fastidiosipila and Proteiniborus.

Temperature yielded 34 discriminating genera. At $23^{\circ} \mathrm{C}$ taxa such as Methanosaeta, Methanobacterium (both important methane-producing genera) were significantly down-regulated and no methanogenic archaea were determined to be up-regulated discriminants under these conditions (Fig 4). Rather, clades of Proteobacteria were significantly up-regulated at the low temperature (Fig 5).

Finally, the deprivation of cobalt under both acetate-supplied and VFA-supplied conditions resulted the down-regulation of a number of methanogenic archaea including Methanosaeta, Methanospirillum, and Methanolinea (Fig 4). However, clades of Bacteroidetes and Synergistetes were significantly up-regulated and presumably unaffected by the lack of cobalt (Fig 5).

\section{Discussion}

\section{The development of individual aggregates}

If we assume, as proposed Díaz et al. [14], that small granules can be considered 'young' and large granules 'old', the comparison of different sizes in this study yield some interesting insights into biofilm development as granules age. The size distribution was statistically similar across the three biomass sources, suggesting: firstly, that in anaerobic bioreactors a range of biofilm sizes can be found, which was also previously observed elsewhere [15]; and secondly, that the ecosystem, and size distribution, in such bioreactors may reach a state of equilibrium, partially controlled by the prevailing hydrodynamic and shear conditions $[16,17]$. Differences in TS and VS across the sizes may also contribute to a 
conceptual model on granule development. Small granules were observed to have the lowest VS, which peaked in medium-sized granules and decreased again in large granules. This may support the granulation theory proposed by Pereboom [17] that granules form as cells attach to inorganic nuclei such that they have relatively less organic material when newly-formed.

Methanogenic activity also shifted across the size fractions. Overall, methane generation rates were higher against $\mathrm{H}_{2} / \mathrm{CO}_{2}$ than acetate, suggesting the predominance of active hydrogenotrophic methanogens. However, with increasing size fraction, activity against acetate increased in granules from all three sources. This may suggest that as granules age, the acetoclastic pathway becomes more important, or that changes in the biofilm structure of larger granules simply allow for increased access to acetate.

Across the sizes, significant differences were observed in the microbiome. Alpha diversity tended to decrease as granule size increased suggesting that as granules age, one or more taxonomic groups become(s) more dominant. This was previously observed [18], but using only one sludge sample. Here the same trend was observed from three different sludge sources indicating that this is likely a common trajectory for microbiome diversity in anaerobic bioreactors. Moreover, it was observed in both cases that a group of methanogenic archaea appeared to be the dominating functional group, supporting the idea that the granules become more anaerobic as they develop [18].

\section{Sludge source shapes community structure}

While significant differences in community structure were observed between the sizes, greater significance was observed according to biomass source. Difference in community structure between various biomass sources was previously reported, and is expected [19-21]. In this study beta diversity clearly shows the three sources clustering independently. Moreover, PERMANOVA verified that source was more significant than size in driving community structure. Just as Batstone and Keller [15] found that wastewater type was the most significant driver of bulk properties of anaerobic granules, it is likely that the different wastewaters on which the three biomass sources were cultivated explains differences in the microbiomes. Indeed, community clustering according to sample source was also previously observed from anaerobic digestate sampled from biogas reactors [22].

MINT analysis identified ten discriminant genera which were responsible for changes across the three sources and also across the three sizes. Interestingly, none of those genera belonged to the methanogenic archaea. This suggests that methanogenic Euryarchaeota belong to a core AD microbiome present across each of the sources and sizes. Indeed, our study suggests that these archaea make up $\sim 50 \%$ of the relative abundance in large granules. Previous studies also identified the Euryarchaeota as a part of the core $A D$ microbiome, although these studies were on anaerobic digestate rather than granules $[22,23]$. In any case, our results suggest that regardless of source or development stage, Euryarchaeota will be abundant in methanogenic granular biofilms. 
The TS and VS of one hundred individual Large granules were statistically similar $(p=0.457$ and 0.635 , respectively) indicating that individual granules have similar and perhaps replicated physical characteristics. Moreover, sequencing of $16 \mathrm{~S}$ rRNA genes from cDNA from 16 individual large granules indicated statistical similarity based on community structure. Previous studies sequenced $16 \mathrm{~S}$ genes of single anaerobic granules from DNA, concluding granules were heterogenic and different granules may perform different functions [13]. While interesting, our study selectively investigated the active community in individual granules finding they are statistically identical, or homogenic and that at the depth of $16 \mathrm{~S}$ rRNA gene sequencing individual granules (from a specific size fraction from the same source) could be considered highly-replicated whole-ecosystems.

Single granules respond reproducibly to environmental cues

Conceptualising anaerobic granules as highly-replicated whole-ecosystems opens doors to highthroughput biofilm studies in microbial ecology. Regardless of the cues applied, the individual granules yielded reproducible responses with respect to changes in structure of the active component of the microbiome. Notably, Methanobacteriacea, demonstrated metabolic versatility, especially under differing $\mathrm{pH}$ and temperature. Moreover, profiling of VFA demonstrated similar metabolisms among eight replicates under each environmental condition. In the same way that Rillig et al. [3] proposed soil aggregates perform as evolutionary incubators containing complex microbial communities of importance to ecology, we propose individual anaerobic granules are much the same, and are whole-ecosystem biofilms. Countless tests could be conducted on this type of unique, niche community. High-throughput experiments could answer a multitude of important ecological questions regarding microbial community strategies for environmental adaptation and community succession, as well as responses to changing climates.

\section{Conclusions}

In this study an entire community of anoxic microorganisms was investigated in the form of anaerobic granules. Size separation of granules into three distinct fractions showed significant differences in size, replicated across three difference sources. This indicated a possible 'development strategy' in granular biofilms, common across disparate systems and suggests scope to investigate a biofilm development model. Biomass source was a stronger differentiating factor to explain community structure variations between samples. Individual granules were highly replicated microbial biofilms and could be exploited as whole ecosystems, producing replicated responses in the active microbiome to various environmental cues. Overall, the study demonstrated that anaerobic granules are complex niche communities, flexibly shifting their active communities in response to environmental pressures.

\section{Methods}

Source of biomass 
Methanogenic granules were sourced from three different anaerobic bioreactors. Source 1, from a fullscale upflow anaerobic sludge bed (UASB) bioreactor treating sugar-rich wastewater from a sweetener factory at $35^{\circ} \mathrm{C}$. Source 2 , was sourced from a full-scale UASB treating potato-processing wastewater $37^{\circ} \mathrm{C}$. Finally, Source 3 , from a lab-scale UASB treating synthetic VFA-rich wastewater at $37^{\circ} \mathrm{C}$.

\section{Size Fractionation, particle size distribution and solids concentrations}

Each sample was size-fractionated using a range of stainless-steel sieves into three size fractions: small $(S ; \varnothing, 0.4-0.8 \mathrm{~mm})$, medium $(\mathrm{M} ; \varnothing, 0.8-1.2 \mathrm{~mm})$, and large $(L ; \varnothing, 1.2-2.0 \mathrm{~mm})$. The size distribution of each source was calculated as the quotient of the average wet weight of individual granules of each size fraction from the total wet weight of each size fraction. TS and VS concentrations were then measured following standard methods [24].

\section{Methanogenic activity assays}

The methanogenic activity of each size fraction from each of the sources was measured using SMA assays $[25,26]$. An SMA buffer was added with methanogenic granules to $60-\mathrm{ml}$ glass bottles to give a final volume of $10 \mathrm{ml}$, and a final VS concentration of $2-5 \mathrm{~g} \mathrm{~L}^{-1}$. The bottles were sealed and $\mathrm{N}_{2}$-flushed before acclimatisation at $37^{\circ} \mathrm{C}$ for $48 \mathrm{~h}$. Aliquots of $0.1 \mathrm{ml}$ soluble substrates were then added to separate, respective bottles to give final concentrations of $30 \mathrm{mM}$ acetate, $15 \mathrm{mM}$ butyrate, or $30 \mathrm{mM}$ propionate. To test for autotrophic methanogenesis, $\mathrm{H}_{2} / \mathrm{CO}_{2}(80: 20, \mathrm{v} / \mathrm{v})$ was added at 1 bar for $20 \mathrm{~s}$. Complimentary control tests were set-up with no substrate addition to measure background activity. Headspace biogas pressure was measured as millivolts (mv), using a handheld pressure transducer (CentrePoint Electronics, Galway, Ireland), and converted to biogas volume $(\mathrm{ml})$ using a headspace correction factor. Gas chromatography (CP-3800, VARIAN, Inc., Walnut Creek, CA) was used to determine the methane concentration (\%) in the biogas, and the accumulation rate was plotted and an activity rate was calculated.

Set-up of micro batch reactors (mBR)

Large granules $(\varnothing, 1.2-2.0 \mathrm{~mm})$ from Source 3 were sampled and single granules were transferred into individual wells in 48-well plates (mBR). Anaerobic basal medium (details in Supplementary Materials) and corresponding substrates were added in $1 \mathrm{ml}$ aliquots to each granule within an anaerobic chamber. mBR were then wrapped in parafilm (Parafilm M Wrapping Film, Fisher Scientific) and incubated for 42 days at either $37^{\circ} \mathrm{C}$ inside an anaerobic box (BD GasPak EZ), or at $23^{\circ} \mathrm{C}$ in the anaerobic chamber. $\mathrm{mBR}$ were operated as 48-h batch incubations, with fresh medium supplied at the start of each cycle.

Eighteen different environmental conditions were tested (Fig 6) as factors which affect growth and activity. These included: (i) the pH effect $(4,7$, and 10$)$; (ii) the temperature effect $\left(37^{\circ} \mathrm{C}\right.$ and $23^{\circ} \mathrm{C}$ ); (iii) the substrate effect (VFA mixture, acetate, cellulose, or glucose); (iv) the cobalt effect (supplied or deprived [27]); and (v) the inhibitor effect (each operated with and without the addition of BES as a methanogenic inhibitor [28]). 
Sugar and VFA concentrations were monitored during the $\mathrm{mBR}$ trial as a means of targeted metabolomic profiling. During the $m B R$ trial, liquor samples $(n=8)$ were collected for each relevant environmental condition: sugars were measured during single granules supplied with cellulose or glucose while VFA were monitored for each environmental condition.

Sugars were measured using the Dubois method [29] with glucose as the standard. VFA profiling was measured using gas chromatography [30]. A flame ion detector (FID) was used and VFA were identified by assigning retention times and spectra to the relevant compounds (acetic, propionic, and butyric acids). Standard VFA calibration curves were used for comparison of relative VFA concentrations and they were expressed as $\mathrm{mg} \mathrm{l}^{-1}$. The internal standard used was 2-Ethylbutyric acid.

\section{DNA/RNA co-extraction and cDNA synthesis}

Genomic DNA and RNA were co-extracted from each sample: (i) from single granules $(n=5)$ from the three sources, each separated into three size fractions, (ii) from single granule replicates $(n=16)$ before treatment, and (iii) from single granules $(n=3)$ from each treatment.

Nucleic acids were co-extracted following the method previously described [31], which is based on bead beating in $5 \%(\mathrm{w} / \mathrm{v})$ cetyl trimethylammonium bromide (CTAB) extraction buffer, followed by phenolchloroform extraction. Integrity of nucleic acids was assessed using a nanodrop (Thermo Fisher Scientific, Waltham, MA, USA). Concentrations were determined using a Qubit fluorometer (Invitrogen, Carlsbad, CA, USA) and normalised to $5 \mathrm{ng}$ DNA $\mu{ }^{-1}$ before storage at $-80^{\circ} \mathrm{C}$.

Subsequent CDNA synthesis from RNA was performed for the 16 single granule replicates with no environmental treatment, and for the single granules from the $\mathrm{mBR}$ trial in triplicate from each condition. Samples were defrosted on ice and RNA purification was achieved through a DNase treatment using the TurboDNase kit (AMBION - Invitrogen, Carlsbad, CA, USA) following the manufacturers recommendations. DNA removal was verified by $16 \mathrm{~S}$ rRNA PCR, using primer pair $338 \mathrm{f}$ and $805 \mathrm{r}$ (details in Supplemental Methods). cDNA was synthesised using a master-mix of $\mathrm{MgCl}_{2}$ (50mM), Random Primer Mix (60 mM; BioLabs, MA, USA) and dNTPs (10mM). M-MuLV reverse transcriptase (Biolabs, MA, USA) was used to catalyse synthesis. Concentrations were determined using a Qubit (Invitrogen, Carlsbad, CA, USA) CDNA was stored at $-20^{\circ} \mathrm{C}$ prior to sequencing.

\section{Amplicon sequencing, bioinformatics and statistical analysis}

Amplification of the 16S rRNA gene sequences for (i) the DNA from granules across the three sources and sizes was performed by The Foundation for the Promotion of Health and Biomedical Research of Valencia Region, FISABIO (Valencia, Spain) using the universal bacterial and archaeal primer set: forward primer $515 \mathrm{~F}$ and reverse primer $806 \mathrm{R}$. Amplification of $16 \mathrm{~S}$ rRNA gene sequences from cDNA from (ii) the 16 single granule replicates prior to treatment and (iii) the single granules sampled from various 
environmental conditions was performed by the Research and Testing Laboratory (Lubbock, Texas), using the same primer set. The resulting amplicon libraries of short inserts were sequenced on the Illumina MiSeq platform. Statistical analyses were performed in $\mathrm{R}$ using the data generated from the bioinformatics. Details are described further in Supplementary Methods. An OTU table was generated for this study by matching the original barcoded reads against clean OTUs (a total of 3,873 OTUs for $n=115$ samples) at $97 \%$ similarity.

\section{List Of Abbreviations}

$\mathrm{mBR}$ - micro batch reactor

$A D$ - anaerobic digestion

BES - 2-bromoethanesulfonate

cDNA - complimentary DNA

CTAB - cetyl trimethylammonium bromide

DNA - deoxyribo nucleic acid

FID - flame ion detector

MINT - multivariate integration

OTU - operational taxonomic unit

RNA - ribo nucleic acid

SMA - specific methanogenic activity

SPLS - sparse projection to latent structure

TS - total solids

UASB - upflow anaerobic sludge bed

VFA - volatile fatty acids

VS - volatile solids

\section{Declarations}

Ethics Approval and Consent to Participate. Not applicable

Consent for Publication. Not applicable 
Availability of Data and Material. The sequencing data from this study are available on the European Nucleotide Archive under the study accession number PRJEB29753. Additional data are provided as supplementary materials.

Competing Interests. The authors declare that they have no competing interests.

Funding. CQ was funded by an MRC fellowship MR/M50161X/1 as part of the CLoud Infrastructure for Microbial Genomics (CLIMB) consortium MR/L015080/1. UZI was funded by NERC IRF NE/L011956/1. GC, SM, SOS and ACT were supported by a European Research Council Starting Grant (3C-BIOTECH 261330) and by a Science Foundation Ireland Career Development Award (17/CDA/4658) to GC. ACT was further supported by a Thomas Crawford Hayes bursary from NUI Galway, and a Short-Term Scientific Mission grant through the EU COST Action 1302.

Authors' Contributions. SOS and GC designed the study with analysis contributions from ACT and UZI. SOS performed all of the physico-chemical characterisation and prepared the sequencing libraries. UZI wrote the scripts for data analysis, which was conducted by ACT. Results were interpreted by ACT, SOS, EPB, SM, CQ, UZI and GC. ACT drafted the paper and UZI and GC revised the document. UZI and GC are joint corresponding authors and SOS and ACT are joint first authors. All authors approve the paper and agree for accountability of the work therein.

Acknowledgements. The authors thank NVP Energy for providing anaerobic sludge granules.

\section{References}

1. Hatzenpichler R, Krukenberg V, Spietz RL, Jay ZJ. Next-generation physiology approaches to study microbiome function at single cell level. Nat Rev Microbiol [Internet]. 2020; Available from: https://doi.org/10.1038/s41579-020-0323-1

2. Cordero OX, Datta MS. Microbial interactions and community assembly at microscales. Curr Opin Microbiol [Internet]. 2016;31:227-34. Available from: http://www.sciencedirect.com/science/article/pii/S1369527416300340

3. Rillig MC, Muller LAH, Lehmann A. Soil aggregates as massively concurrent evolutionary incubators. Isme J [Internet]. The Author(s); 2017;11:1943. Available from: http://dx.doi.org/10.1038/ismej.2017.56

4. van Lier JB, van der Zee FP, Frijters CTMJ, Ersahin ME. Celebrating 40 years anaerobic sludge bed reactors for industrial wastewater treatment. Rev Environ Sci Bio/Technology [Internet]. 2015;14:681-702. Available from: https://doi.org/10.1007/s11157-015-9375-5

5. Milferstedt K, Hamelin J, Park C, Jung J, Hwang Y, Cho S-K, et al. Biogranules applied in environmental engineering. Int J Hydrogen Energy [Internet]. 2017;42:27801-11. Available from: http://www.sciencedirect.com/science/article/pii/S0360319917330124

6. Wilbanks EG, Jaekel U, Salman V, Humphrey PT, Eisen JA, Facciotti MT, et al. Microscale sulfur cycling in the phototrophic pink berry consortia of the Sippewissett Salt Marsh. Environ Microbiol 
[Internet]. 2014;16:3398-415. Available from: https://doi.org/10.1111/1462-2920.12388

7. Togashi T, Sasaki H, Yoshimura J. A geometrical approach explains Lake Ball (Marimo) formations in the green alga, Aegagropila linnaei. Sci Rep [Internet]. The Author(s); 2014;4:3761. Available from: https://doi.org/10.1038/srep03761

8. Leng L, Yang P, Singh S, Zhuang H, Xu L, Chen W-H, et al. A review on the bioenergetics of anaerobic microbial metabolism close to the thermodynamic limits and its implications for digestion applications. Bioresour Technol. Elsevier; 2018;247:1095-106.

9. Hulshoff Pol LW, de Castro Lopes SI, Lettinga G, Lens PNL. Anaerobic sludge granulation. Water Res [Internet]. 2004;38:1376-89. Available from:

http://www.sciencedirect.com/science/article/pii/S0043135403006705

10. Gujer W, Zehnder AJB. Conversion Processes in Anaerobic Digestion. Water Sci Technol [Internet]. 1983;15:127. Available from: http://dx.doi.org/10.2166/wst.1983.0164

11. Campanaro S, Treu L, Kougias PG, De Francisci D, Valle G, Angelidaki I. Metagenomic analysis and functional characterization of the biogas microbiome using high throughput shotgun sequencing and a novel binning strategy. Biotechnol Biofuels [Internet]. 2016;9:26. Available from: https://doi.org/10.1186/s13068-016-0441-1

12. Leventhal GE, Boix C, Kuechler U, Enke TN, Sliwerska E, Holliger C, et al. Strain-level diversity drives alternative community types in millimetre-scale granular biofilms. Nat Microbiol. Nature Publishing Group; 2018;3:1295.

13. Kuroda K, Nobu MK, Mei R, Narihiro T, Bocher BTW, Yamaguchi T, et al. A Single-Granule-Level Approach Reveals Ecological Heterogeneity in an Upflow Anaerobic Sludge Blanket Reactor. PLoS One [Internet]. Public Library of Science; 2016;11:e0167788. Available from: https://doi.org/10.1371/journal.pone.0167788

14. Díaz EE, Stams AJM, Amils R, Sanz JL. Phenotypic properties and microbial diversity of methanogenic granules from a full-scale upflow anaerobic sludge bed reactor treating brewery wastewater. Appl Environ Microbiol. 2006;72:4942-9.

15. Batstone DJ, Keller J. Variation of bulk properties of anaerobic granules with wastewater type. Water Res [Internet]. 2001;35:1723-9. Available from: http://www.sciencedirect.com/science/article/pii/S0043135400004462

16. Arcand $Y$, Guiot SR, Desrochers $M$, Chavarie $C$. Impact of the reactor hydrodynamics and organic loading on the size and activity of anaerobic granules. Chem Eng J Biochem Eng J [Internet]. 1994;56:B23-35. Available from: http://www.sciencedirect.com/science/article/pii/0923046794870284

17. Pereboom JHF. Size distribution model for methanogenic granules from full scale UASB and IC reactors. Water Sci Technol. 1994;30:211-21.

18. Trego AC, Morabito C, Bourven I, Guibaud G, Mills S, Connelly S, et al. Diversity Converges During Community Assembly In Methanogenic Granules, Suggesting A Biofilm Life-Cycle. bioRxiv [Internet]. 
Cold Spring Harbor Laboratory; 2018; Available from:

https://www.biorxiv.org/content/early/2018/12/04/484642

19. Leclerc $M$, Delgènes J, Godon J. Diversity of the archaeal community in 44 anaerobic digesters as determined by single strand conformation polymorphism analysis and 16S rDNA sequencing. Environ Microbiol. Wiley Online Library; 2004;6:809-19.

20. Li J, He S, Wang X, Xia S, Duan L. Microbial Community Structures in Two Membrane Bioreactors: Sequential and Continuous Modes. 2010 4th Int Conf Bioinforma Biomed Eng. IEEE; 2010. p. 1-4.

21. Bialek K, Kumar A, Mahony T, Lens PNL, O'Flaherty V. Microbial community structure and dynamics in anaerobic fluidized-bed and granular sludge-bed reactors: influence of operational temperature and reactor configuration. Microb Biotechnol. Wiley Online Library; 2012;5:738-52.

22. Calusinska M, Goux X, Fossépré M, Muller EEL, Wilmes P, Delfosse P. A year of monitoring 20 mesophilic full-scale bioreactors reveals the existence of stable but different core microbiomes in bio-waste and wastewater anaerobic digestion systems. Biotechnol Biofuels [Internet]. 2018;11:196. Available from: https://doi.org/10.1186/s13068-018-1195-8

23. Campanaro S, Treu L, Kougias PG, Luo G, Angelidaki I. Metagenomic binning reveals the functional roles of core abundant microorganisms in twelve full-scale biogas plants. Water Res [Internet]. 2018;140:123-34. Available from:

http://www.sciencedirect.com/science/article/pii/S0043135418303373

24. APHA. Standard methods for the examination of water and wastewater. 21 st ed. New York: American Public Health Association, Washington DC; 2005.

25. Colleran E, Concannon F, Golden T, Geoghegan F, Crumlish B, Killilea E, et al. Use of Methanogenic Activity Tests to Characterize Anaerobic Sludges, Screen for Anaerobic Biodegradability and Determine Toxicity Thresholds against Individual Anaerobic Trophic Groups and Species. Water Sci Technol [Internet]. 1992;25:31 LP - 40. Available from: http://wst.iwaponline.com/content/25/7/31.abstract

26. Coates JD, Coughlan MF, Colleran E. Simple method for the measurement of the hydrogenotrophic methanogenic activity of anaerobic sludges. J Microbiol Methods [Internet]. 1996;26:237-46. Available from: http://www.sciencedirect.com/science/article/pii/0167701296009153

27. Chen JL, Steele TWJ, Stuckey DC. Stimulation and Inhibition of Anaerobic Digestion by Nickel and Cobalt: A Rapid Assessment Using the Resazurin Reduction Assay. Environ Sci Technol [Internet]. American Chemical Society; 2016;50:11154-63. Available from: https://doi.org/10.1021/acs.est.6b03522

28. Zinder SH, Anguish T, Cardwell SC. Selective inhibition by 2-bromoethanesulfonate of methanogenesis from acetate in a thermophilic anaerobic digestor. Appl Environ Microbiol. Am Soc Microbiol; 1984;47:1343-5.

29. DuBois M, Gilles KA, Hamilton JK, Rebers PA, Smith F. Colorimetric Method for Determination of Sugars and Related Substances. Anal Chem [Internet]. American Chemical Society; 1956;28:350-6. Available from: https://doi.org/10.1021/ac60111a017 
30. Nzeteu CO, Trego AC, Abram F, O'Flaherty V. Reproducible, high-yielding, biological caproate production from food waste using a single-phase anaerobic reactor system. Biotechnol Biofuels [Internet]. BioMed Central; 2018;11:108. Available from: https://www.ncbi.nlm.nih.gov/pubmed/29651303

31. Griffiths RI, Whiteley AS, O'Donnell AG, Bailey MJ. Rapid Method for Coextraction of DNA and RNA from Natural Environments for Analysis of Ribosomal DNA- and rRNA-Based Microbial Community Composition. Appl Environ Microbiol [Internet]. American Society for Microbiology; 2000;66:5488-91. Available from: http://www.ncbi.nlm.nih.gov/pmc/articles/PMC92488/

\section{Additional Files}

Additional File 1: Supplemental materials and methods

Additional File 2: Figures S1: Physiological characteristics of granules separated by size; Figure S2; Metabolic profiles of single granules; and Figure S3 Sugar and VFA profiles of single granules.

\section{Figures}



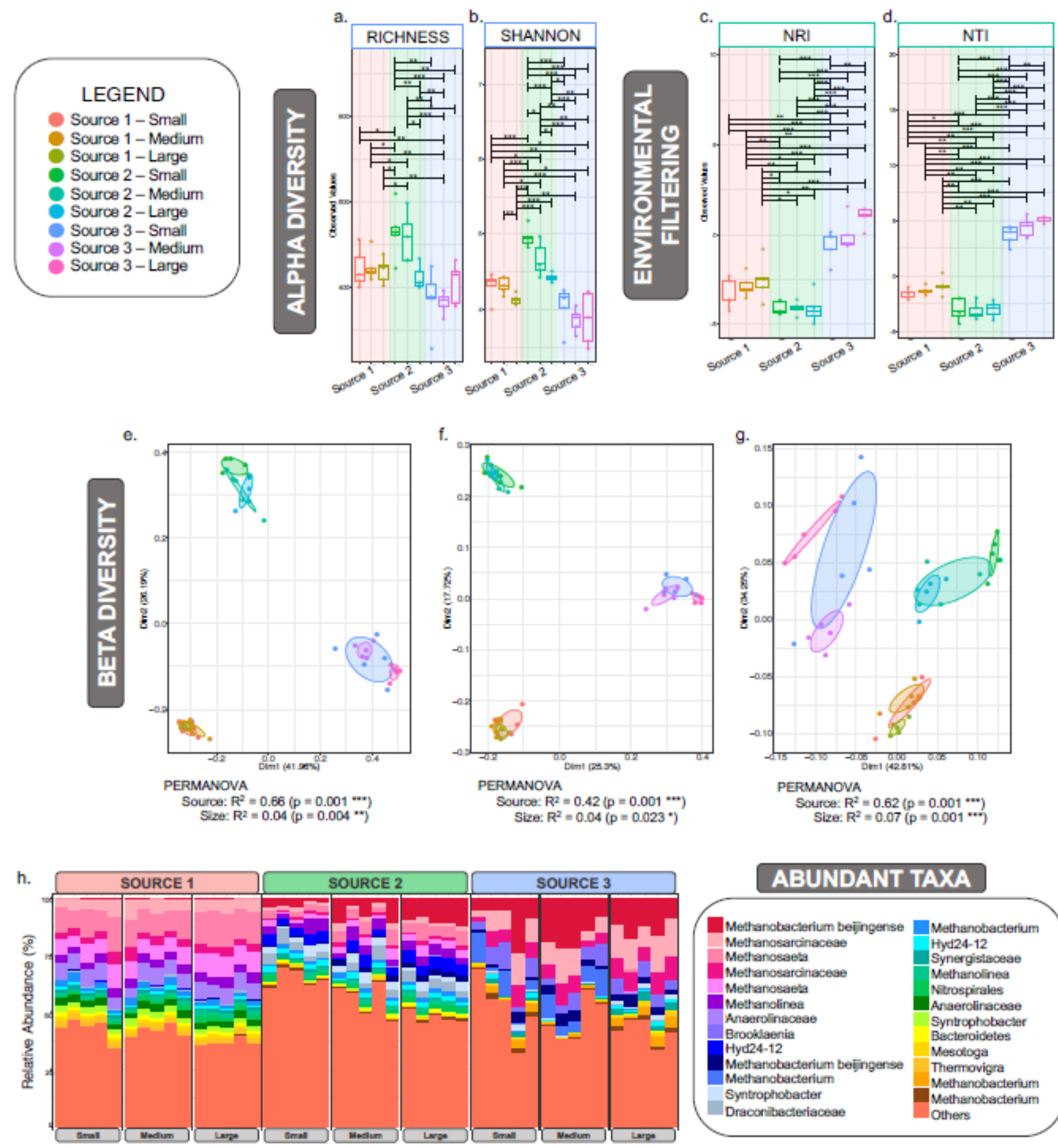

\section{ABUNDANT TAXA}

Methanobacterium beīingense Methanobacterium Methanosarcinaceae Methanosaeta

Methanosarcinaceae

Methanosaeta

Methanolinea

Anaerolinaceae

Brooklaenia

Hyd 24-12

Methanobacterium beịngense

Syntrophobacter

Dracon Bacteriaceae

Nethanobacterium
Hyd24-12
Synergistaceae
Nethanolines
Nitrospirales
Anaerolinaceae
Syntrophobacter
Bacteroidetes
Mesotoga
Thermovigra
Methanobacterium
Methanobacterium
Others

Figure 1

Microbial diversity and community structure according to variances in the 16S rRNA gene in DNA from single granules $(n=5)$ from three community sources and across three discrete sizes (small, medium, and large). Alpha diversity: box plot of the (a) rarefied species richness and (b) Shannon Entropy; Environmental Filtering: (c) Net Relatedness Index (NRI) and (d) Nearest Taxa Index (NTI) calculated using the phylogenetic tree with presence/absence abundance; Beta diversity: Non-Metric Multidimensional Scaling (NMDS) using (e) Bray-Curtis dissimilarity ( $f$ ) UniFrac; and (g) weighted UniFrac 
distances, where each point corresponds to the community structure of one sample, size fractions are indicated by colour, the ellipses are drawn at a $95 \% \mathrm{Cl}$, and PERMANOVA statistics shown beneath the plots; (h) community structure based on relative abundance of the top-25 most abundant OTUs from across each size fraction, where 'others' refers to all OTUs not included in the 'top-25'; lines for figures $a, b$, $c \& d$ connect two categories where the differences were significant (ANOVA) with * $(P<0.05)$, ** $(P<$ $0.01)$, or $* \star \star(p<0.001)$.
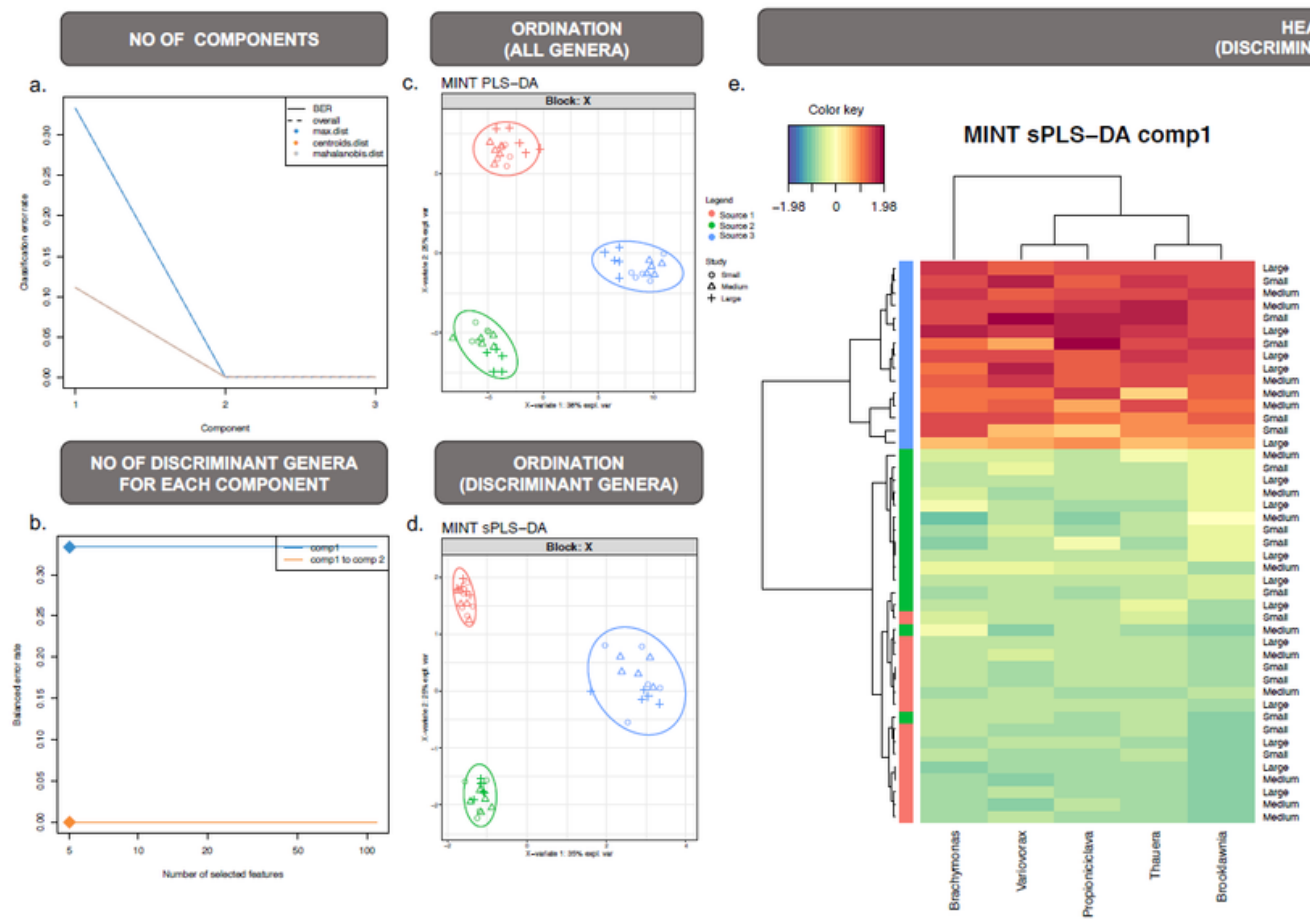

HEATMAP
MINANT GENERA)

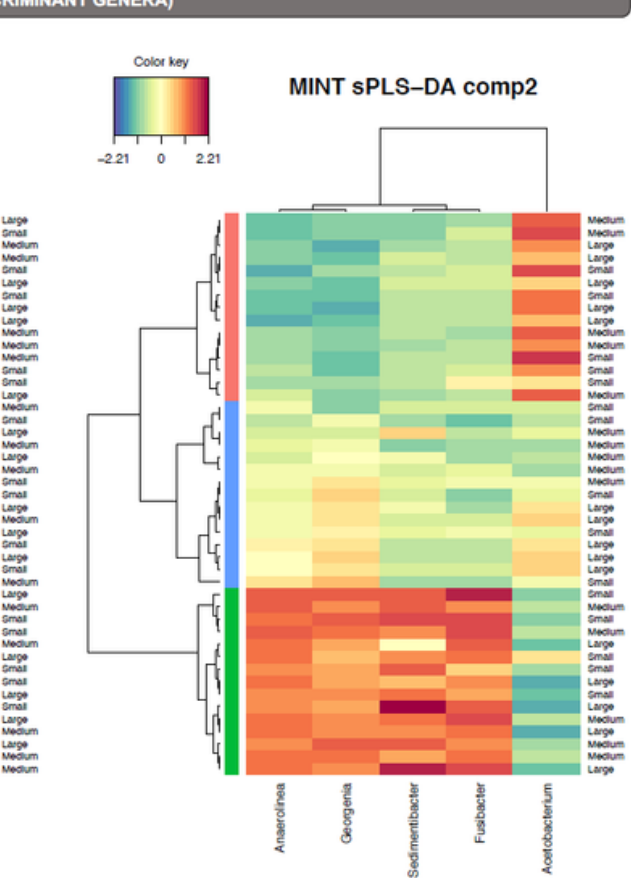

Figure 2

MINT study-wise discriminant analysis is a two-step process where (a) we found two components that reduce the classification error rates significantly using centroids distance in the algorithm; in step two, (b) we found 5 discriminating genera from both components; (c) shows the ordination of samples using all the genera in the two components (MINT PLS-DA) with ellipse representing $95 \%$ confidence interval and percentage variations explained by these components in axes labels; in (d) samples are drawn only using the discriminants from the two components (MINT sPLS-DA); (e) shows the MINT sPLS heatmaps of the discriminant genera, with both rows and columns ordered using hierarchical (average linkage) clustering to identify blocks of genera of interest. Heatmap depicts TSS+CLR normalised abundances: high abundance (red) and low abundance (blue). 


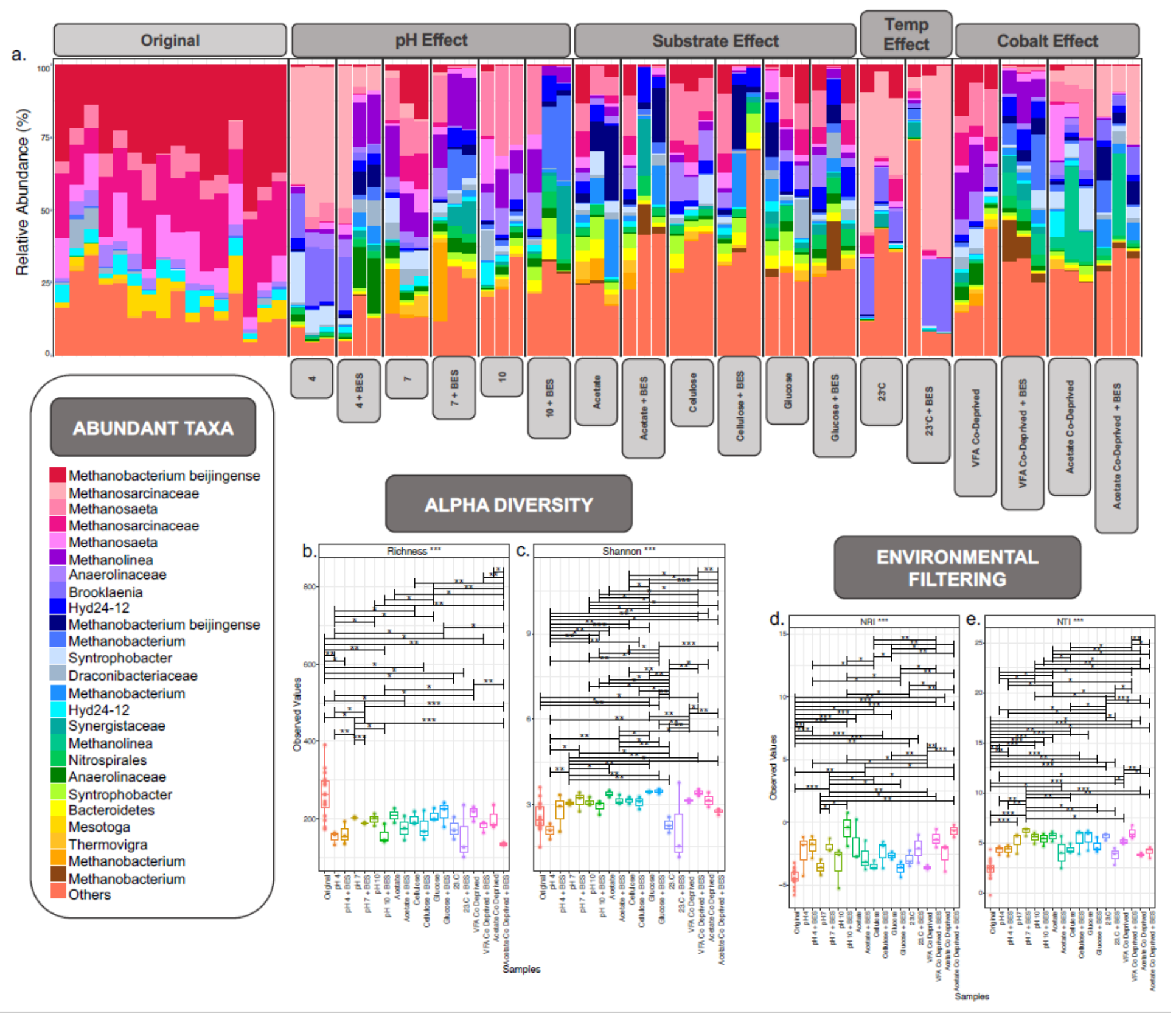

\section{Figure 3}

Active microbial diversity and community structure according to variances in the 16S rRNA gene from single granule, whole-ecosystems from untreated, original $(n=16)$ granules, as well as granules $(n=3)$ treated under various, $\mathrm{pH}$, substrate, temperature and cobalt deprivation effects where (a) shows the community structure based on relative abundance of the top-25 most abundant OTUs from across each size fraction, where 'others' refers to all OTUs not included in the 'top-25'; (b) box plot of the rarefied species richness and (c) Shannon Entropy; Environmental Filtering depicted as the (d) Net Relatedness Index (NRI) and (e) Nearest Taxa Index (NTI) calculated using the phylogenetic tree with presence/absence abundance; lines for figures b, $c, d, \&$ e connect two categories where the differences

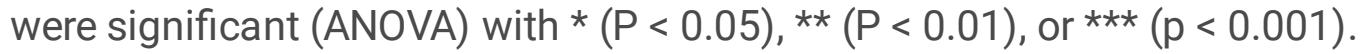



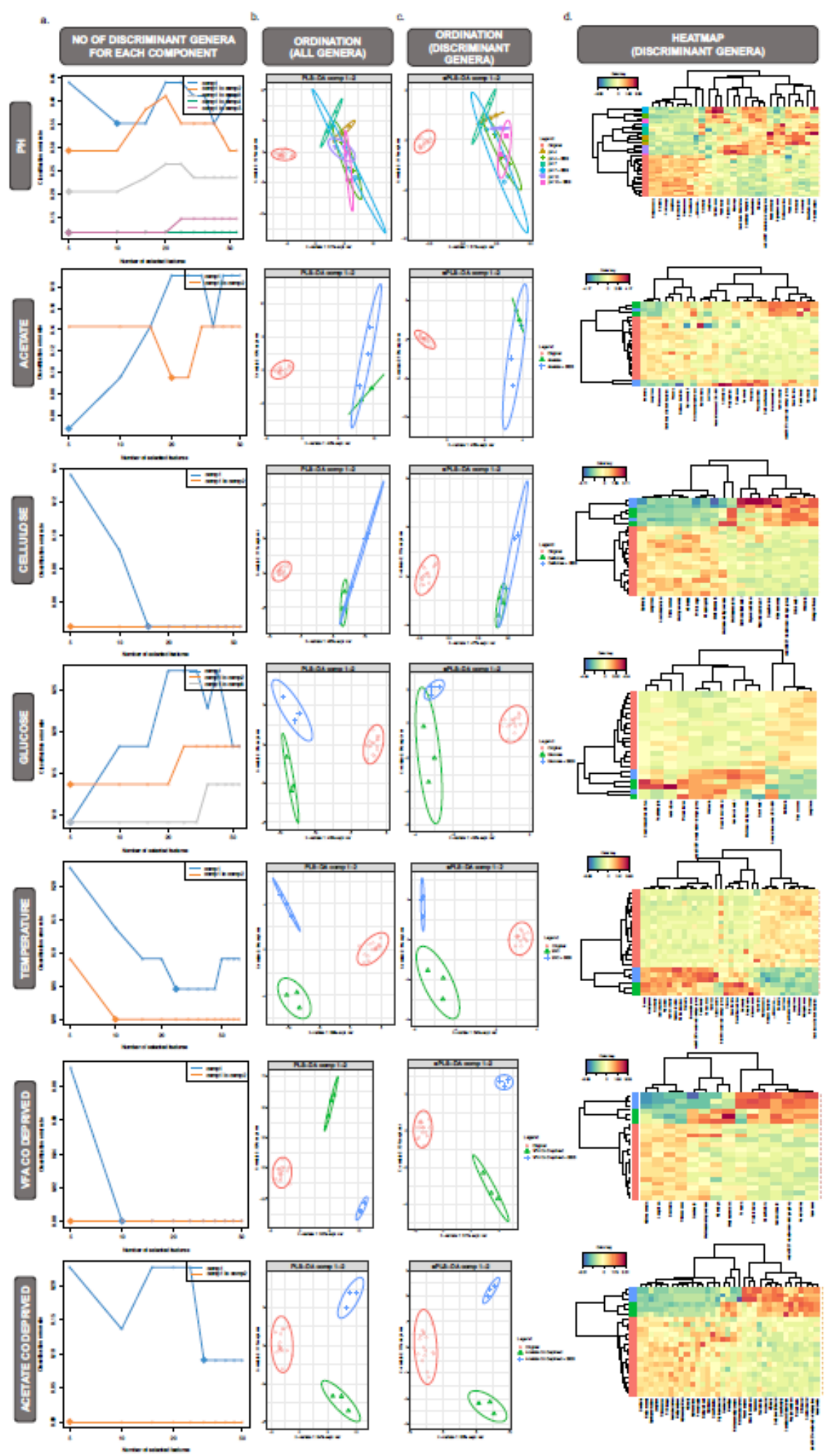

\section{Figure 4}

sPLS-DA discriminant analysis showing (a) the number of discriminant genera found for each component; (b) the ordination of samples using all the genera in the first two components (sPLS-DA) with ellipse representing 95\% confidence interval and percentage variations explained by these components in axes labels; (c) the discriminants from the two components (sPLS-DA); and (d) the sPLS-DA heatmaps of all the discriminant genera, with both rows and columns ordered using hierarchical (average linkage) 
clustering to identify blocks of genera of interest. Heatmap depicts TSS+CLR normalised abundances: high abundance (red) and low abundance (blue).
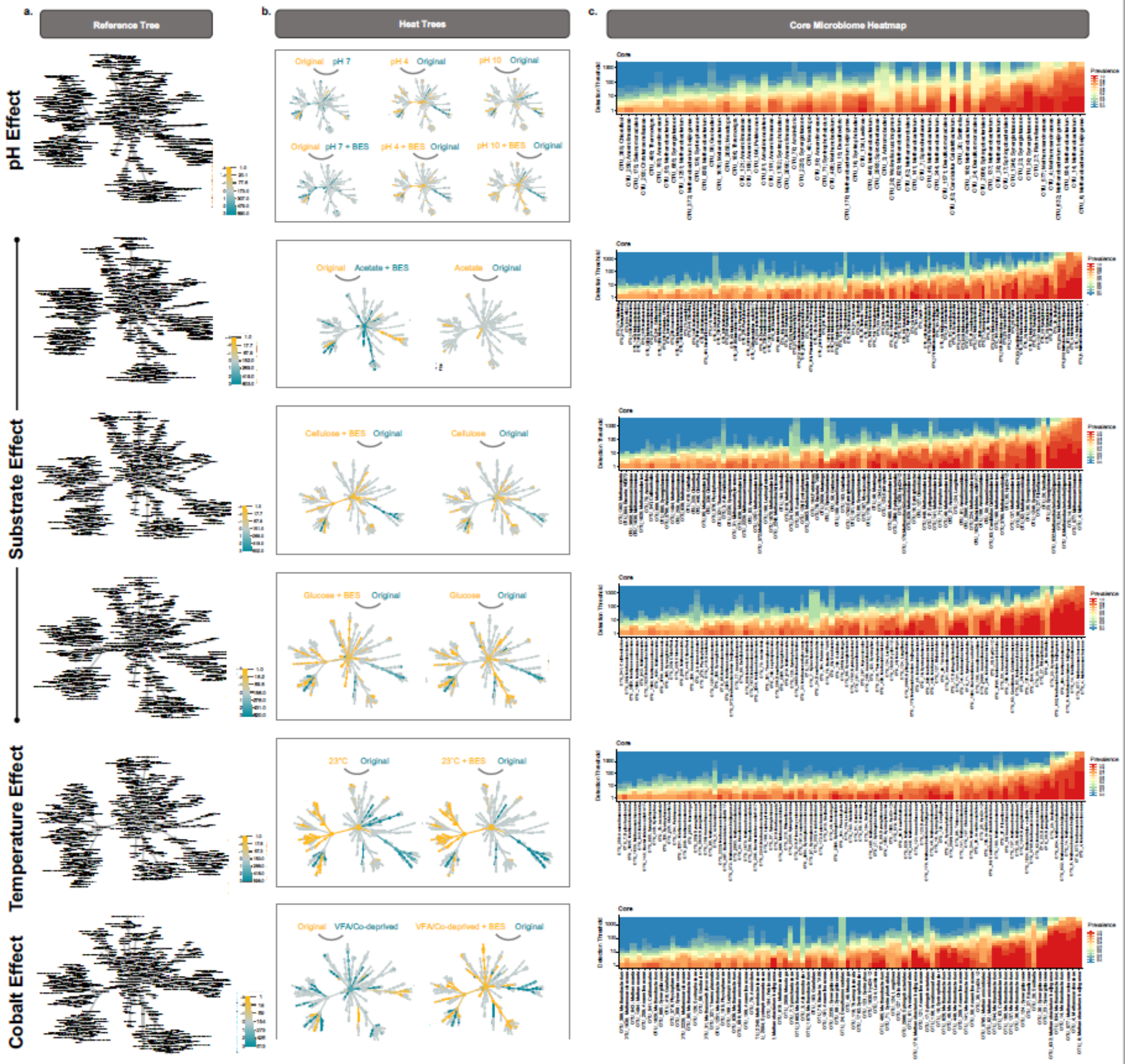

\section{Figure 5}

Dynamic (differential heat tree) vs. Persistant (core microbiome) components of single-granule, whole microbial communities subjected to various environmental conditions where (a) shows a reference tree; (b) shows the differential heat tree of clades that are up/down-regulated between multiple categories, where the color represents the condition wherein they are dominant; and (c) is a heatmap showing the 
core microbiome at a minimum of $75 \%$ prevalence, with the $y$-axis representing the detection threshold at different abundances. The OTUs are sorted based on increasing order of abundances.

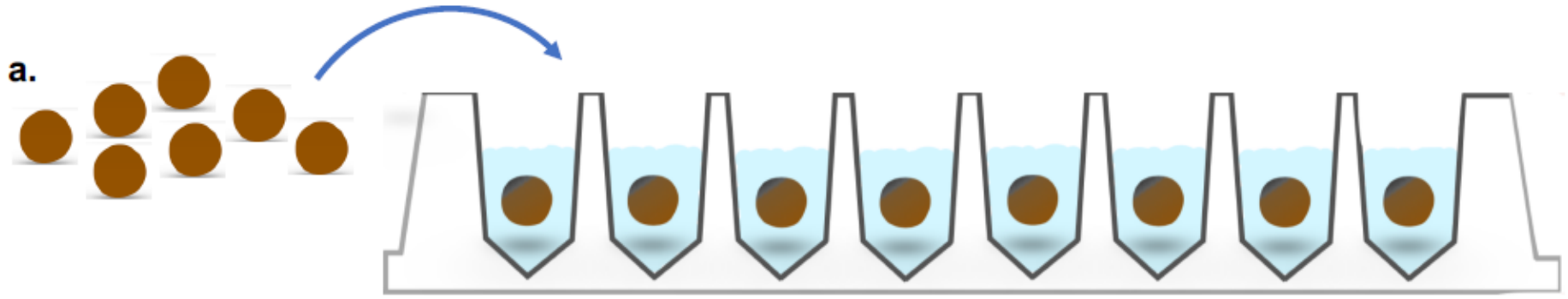

b.

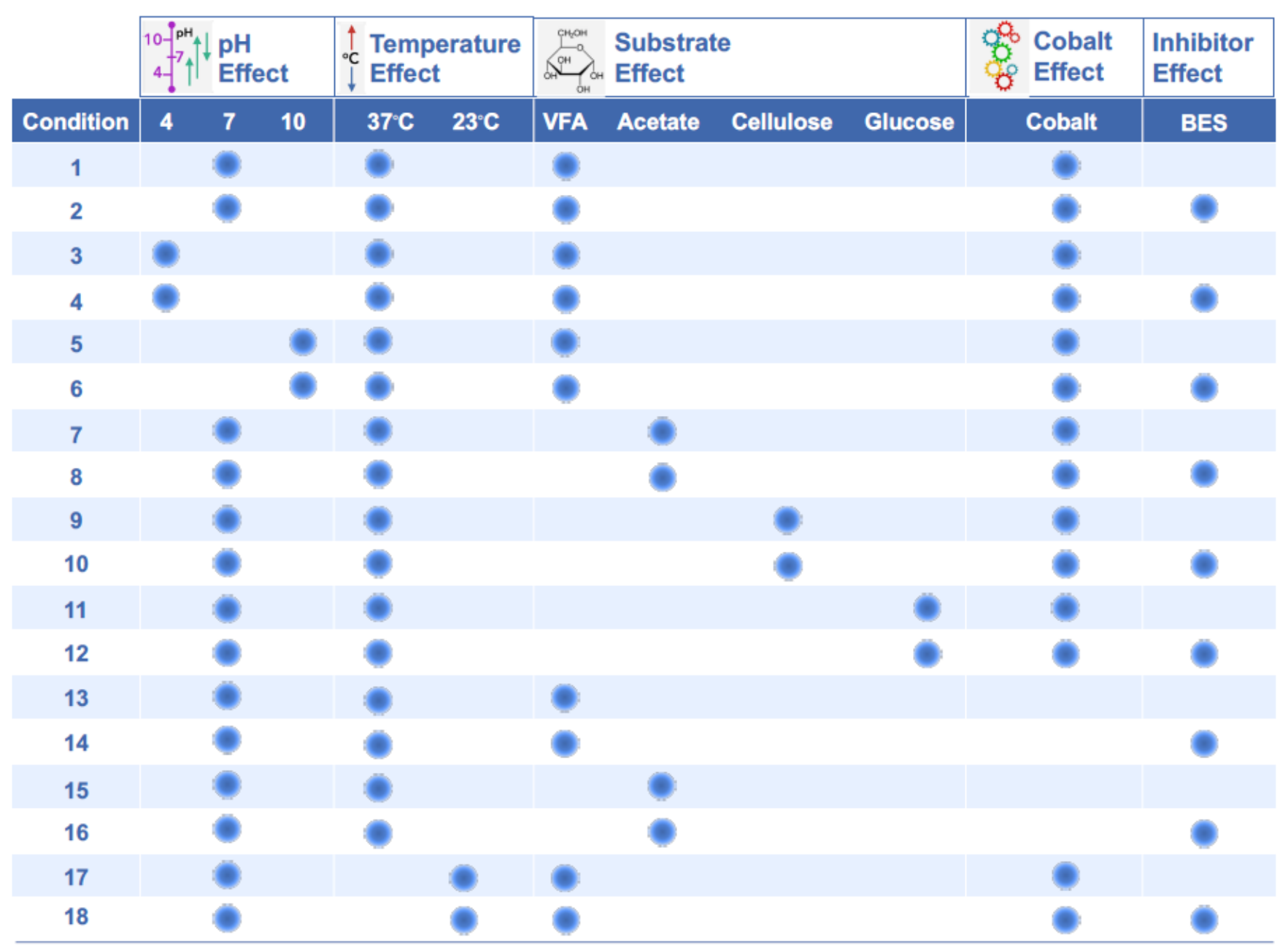

\section{Figure 6}

Single granules, representing whole-ecosystems from one source and one size fraction, were placed into different wells of 48-well plates (a) and incubated under different environmental conditions (b) in a highthroughput investigation into the impact of environmental cues on whole microbiomes

\section{Supplementary Files}

This is a list of supplementary files associated with this preprint. Click to download. 
- TregoetalMicrobiomeGraphicalAbstractPDF.pdf

- TregoetalMicrobiomeAdditionalFile2.pdf

- TregoetalMicrobiomeAdditionalFile1.pdf 